\title{
WICK-TYPE STOCHASTIC KdV EQUATION BASED ON LÉVY WHITE NOISE
}

\author{
ABD-ALLAH HYDER \\ Department of Engineering Mathematics and Physics \\ Faculty of Engineering \\ Al-Azhar University \\ Cairo \\ Egypt \\ e-mail: abdallah.hyder@yahoo.com
}

\begin{abstract}
Wick-type stochastic KdV equation based on Lévy white noise is researched. Lévy white noise functional solutions are showed by using $F$-expansion method and Hermite transform in Lévy white noise space. These solutions are classified as Jacobi elliptic function and soliton-like solutions. Some of the obtained soliton-like solutions are sketched graphically.
\end{abstract}

\section{Introduction}

It is well known that random waves are important subject of stochastic partial differential equations (SPDEs). There were many researchers studied this subject. In [1], Wadati first introduced and studied the stochastic KdV equation and gave the diffusion of the soliton solution of the $\mathrm{KdV}$ equation under Gaussian white noise. The Wick-type stochastic KdV equation on the Gaussian white noise space was first 2010 Mathematics Subject Classification: 05.40.Ca, 02.30.Jr. Keywords and phrases: KdV equation, Lévy white noise, Wick-type, $F$-expansion method. Submitted by Zhuangui Liu. Received February 22, 2017

(C) 2017 Scientific Advances Publishers 
introduced by Xie in [2], he showed the Bäcklund transformation and the exact white noise functional solutions by using the homogeneous balance principle. Furthermore, Chen and Xie [3-6], Xie [7-9], Ghany [10-12], Ghany et al. [13], and Ghany and Hyder [14-18] investigated some stochastic travelling wave equations using Gaussian white noise analysis. On the other hand, an extension of Gaussian white noise analysis to non-Gaussian white noise analysis was established in [21], and developed further in [22, 23]. Based on this extension, Løkka et al. [24] and Øksendal [25] developed a white noise framework for the study of SPDEs driven by a $d$-parameter Lévy white noise, which is in fact a non-Gaussian white noise. Recently, Hyder and Zakarya [20] have developed a non-Gaussian Wick calculus based on the theory of hypercomplex systems $L_{1}(Q, d m(x))$. Using the Delsarte characters $\chi_{n}(x)$, they introduced a $\chi$-Wick product, a $\chi$-Hermite transform on the space of generalized functions $H_{-q}^{\chi}$ and setup a framework to study the stochastic partial differential equations driven by $H_{-q}^{\chi}$-processes. Moreover, Ghany et al. [19] applied this framework and $F$-expansion method and gave non-Gaussian white noise functional solutions of $\chi$-Wick-type stochastic KdV equations.

The main objective of this paper is to use the theory introduced in [24-26] to study a Wick-type analogue of the KdV equation, constructed by means of the Lévy measure. Precisely, we give exact white noise functional solutions of Wick-type stochastic KdV equation based on Lévy white noise, which has the general form

$$
U_{t}+f(t) U \diamond U_{x}+g(t) U_{x x x}=\dot{\eta}(t) \diamond R^{\diamond}\left(U, U_{x}, U_{x x x}\right),
$$

where " $\diamond$ " is the Lévy Wick product on the Kondratiev space $(\mathcal{S})_{-1}$, namely, $(\mathcal{S})_{-1}$ is the Lévy white noise functional space which is defined in the next section. Equation (1.1) is a special stochastic perturbation of the KdV equation with variable coefficients 


$$
u_{t}+f(t) u u_{x}+g(t) u_{x x x}=0
$$

by random force $\dot{\eta}(t) \diamond R^{\diamond}\left(U, U_{x}, U_{x x x}\right)$, where $f(t)$ and $g(t)$ are bounded measurable or integrable functions on $\mathbb{R}_{+}, \dot{\eta}(t)$ is the Lévy white noise, i.e., $\dot{\eta}(t)$ is the time derivative of Lévy process $\eta(t)$, $R\left(u, u_{x}, u_{x x x}\right)=-\alpha u u_{x}-\beta u_{x x x}$ is a functional of $u, u_{x}$, and $u_{x x x}$ for some constants $\alpha, \beta$, and $R^{\diamond}$ is the Wick version of the functional $R$. As pointed out in [1, 27], the motion of long, unidirectional, weakly nonlinear water waves on a channel can be described by Equation (1.2). Moreover, Wang and Wang [28] gave the exact solutions of Equation (1.1) by using the homogeneous balance principle.

In recent years, large amounts of effort have been directed towards finding exact solutions, particularly exact travelling wave solutions, of non-linear partial differential equations. Many powerful methods have been proposed, such as inverse scattering method [29], BäcklundDarboux transformation, $F$-expansion method [30] and so on.

Our first interest in this work is to implement new strategies that give exact solutions of the Wick-type stochastic $\mathrm{KdV}$ equation based on Lévy white noise. The strategies that will be pursued in this work rest mainly on Lévy Hermite transform, Lévy white noise theory, and $F$-expansion method, all of which are employed to find exact Lévy white noise functional solutions of Equation (1.1). The proposed schemes, as we believe, are entirely new and introduce new solutions in addition to the well-known traditional solutions. The ease of using these methods shows its power to determine shock or solitary type of solutions.

\section{Some Basic Concepts about SPDEs Driven by Lévy White Noise}

Assume that the Lévy measure $v$ satisfies the following integrability condition: For all $\epsilon>0$, there exists $\lambda>0$ such that

$$
\int_{|\theta| \geq \epsilon} \exp (\lambda|\theta|) \nu(d \theta)<\infty
$$


This implies that $v$ has finite moments of order $n$ for all $n \geq 2$. Let $\left\{\ell_{m}\right\}_{m \geq 0}$ be the orthogonalization of $\left\{1, \theta, \theta^{2}, \ldots\right\}$ with respect to the inner product of $L^{2}(\rho)$, where $d \rho(\theta):=\theta^{2} \nu(d \theta)$. Then, define the polynomials

$$
p_{m}(\theta):=\frac{\theta \ell_{m-1}(\theta)}{\left\|\ell_{m-1}\right\|_{L^{2}(\rho)}}, \quad m \geq 1
$$

The polynomials $\left\{p_{m}\right\}_{m \geq 1}$ form an orthonormal basis for $L^{2}(\nu)$ (see [31]).

Let $h_{n}(x)$ be the $n$-order Hermite polynomials. Put $\xi_{n}(x)=e^{-\frac{1}{2} x^{2}} h_{n}$ $(\sqrt{2 x}) /(\pi(n-1) !)^{\frac{1}{2}} ; n \geq 1, x \in \mathbb{R}$. We have that the collection $\left\{\xi_{n}\right\}_{n \geq 1}$ constitutes an orthogonal basis for $L^{2}(\mathbb{R})$. If we denote $\alpha=\left(\alpha_{1}, \ldots, \alpha_{d}\right)$ being $d$-dimensional multi-indices with $\alpha_{1}, \ldots, \alpha_{d} \in \mathbb{N}$, we have that the family of tensor products $\xi_{\alpha}=\xi_{\left(\alpha_{1}, \ldots, \alpha_{d}\right)}=\xi_{\alpha_{1}} \otimes \ldots \otimes \xi_{\alpha_{d}} ; \alpha \in \mathbb{N}^{d}$ forms an orthogonal basis for $L^{2}\left(\mathbb{R}^{d}\right)$. Let $\alpha^{(i)}=\left(\alpha_{1}^{(i)}, \ldots, \alpha_{d}^{(i)}\right)$ be the $i$-th multi-index number in some fixed ordering of all $d$-dimensional multi-indices $\alpha=\left(\alpha_{1}, \ldots, \alpha_{d}\right) \in \mathbb{N}^{d}$. We can, and will, assume that this ordering has the property that $i<j \Rightarrow \alpha_{1}^{(i)}+\alpha_{2}^{(i)}+\ldots+\alpha_{d}^{(i)} \leq \alpha_{1}^{(j)}+\alpha_{2}^{(j)}$ $+\ldots+\alpha_{d}^{(j)}$.

To simplify the notation, we write from now on

$$
\xi_{i}(x):=\xi_{\alpha^{(i)}}(x)=\xi_{\alpha_{1}^{(i)}} \otimes \ldots \otimes \xi_{\alpha_{d}^{(i)}} ; \quad i \geq 1, x \in \mathbb{R}^{d} .
$$

Define the bijective map $\kappa: \mathbb{N} \times \mathbb{N} \rightarrow \mathbb{N}$ by

$$
\kappa(i, j)=j+(i+j-2)(i+j-1) / 2 .
$$

Then if $k=\kappa(i, j)$, we define

$$
\delta_{\kappa}(x, \theta)=\delta_{\kappa(i, j)}(x, \theta)=\xi_{i}(x) p_{j}(\theta) ; \quad(i, j) \in \mathbb{N} \times \mathbb{N},(x, \theta) \in \mathbb{R}^{d} \times \mathbb{R} .
$$


We denote multi-indices as elements of the space $\left(\mathbb{N}_{0}^{\mathbb{N}}\right)_{c}$ of all sequences $\alpha=\left(\alpha_{1}, \alpha_{2}, \ldots\right)$ with elements $\alpha_{i} \in \mathbb{N}_{0}$ and with compact support, i.e., with only finitely many $\alpha_{i} \neq 0$. We define $\mathcal{J}=\left(\mathbb{N}_{0}^{\mathbb{N}}\right)_{c}$. Further, we set $\operatorname{Index}(\alpha)=\max \left\{i: \alpha_{i} \neq 0\right\}$ and $|\alpha|=\sum_{i} \alpha_{i}$ for $\alpha \in \mathcal{J}$.

Now, assume $\operatorname{Index}(\alpha)=j$ and $|\alpha|=m$ for $\alpha \in \mathcal{J}$ and identify the function $\delta^{\otimes \alpha}$ as

$$
\begin{aligned}
& \delta^{\otimes \alpha}\left(\left(x_{1}, \theta_{1}\right), \ldots,\left(x_{m}, \theta_{m}\right)\right)=\delta_{1}^{\otimes \alpha_{1}} \otimes \ldots \otimes \delta_{j}^{\otimes \alpha_{j}}\left(\left(x_{1}, \theta_{1}\right), \ldots,\left(x_{m}, \theta_{m}\right)\right) \\
= & \delta_{1}\left(x_{1}, \theta_{1}\right) \ldots \delta_{1}\left(x_{\alpha_{1}}, \theta_{\alpha_{1}}\right) \ldots \delta_{j}\left(x_{\alpha_{1}+\ldots+\alpha_{j-1}+1}, \theta_{\alpha_{1}+\ldots+\alpha_{j-1}+1}\right) \ldots \delta_{j}\left(x_{m}, \theta_{m}\right),
\end{aligned}
$$

where the terms with zero-components $\alpha_{i}$ are set equal to 1 in the product $\left(\delta_{i}^{\otimes 0}=1\right)$. Also, we define the symmetrized tensor product of the $\delta_{k}$ 's denoted by $\delta^{\hat{\otimes} \alpha}$ as

$$
\begin{aligned}
\delta^{\widehat{\otimes} \alpha}\left(\left(x_{1}, \theta_{1}\right), \ldots,\left(x_{m}, \theta_{m}\right)\right) & =\widehat{\delta^{\otimes \alpha}}\left(\left(x_{1}, \theta_{1}\right), \ldots,\left(x_{m}, \theta_{m}\right)\right) \\
& =\delta_{1}^{\widehat{\otimes} \alpha_{1}} \widehat{\otimes} \ldots \widehat{\otimes} \delta_{j}^{\hat{\otimes} \alpha} \alpha_{j}\left(\left(x_{1}, \theta_{1}\right), \ldots,\left(x_{m}, \theta_{m}\right)\right),
\end{aligned}
$$

where “?" denotes symmetrization. For $\alpha \in \mathcal{J}$, we define the random variable

$$
K_{\alpha}=K_{\alpha}(\omega)=I_{|\alpha|}\left(\delta^{\widehat{\otimes}}\right)(\omega) ; \quad \omega \in \mathcal{S}^{\prime}\left(\mathbb{R}^{d}\right),
$$

where $I_{|\alpha|}$ is the iterated integral of order $m=|\alpha|$ with respect to the compensated jump measure of $\eta($.$) , and \mathcal{S}^{\prime}\left(\mathbb{R}^{d}\right)$ is the space of tempered distributions [26]. 
For a fixed $n \in \mathbb{N}$, letting the space $(\mathcal{S})_{1}^{n}$ is composed of those $f(\omega)=$ $\sum_{\alpha} c_{\alpha} K_{\alpha}(\omega) \in \bigoplus_{k=1}^{n} L^{2}(\mu)$ with $c_{\alpha} \in \mathbb{R}^{n}$ such that $\|f\|_{1, k}^{2}=\sum_{\alpha} c_{\alpha}^{2}(\alpha !)^{2}$ $(2 \mathbb{N})^{k \alpha}<\infty, \forall k \in \mathbb{N}$ with $c_{\alpha}^{2}=\left|c_{\alpha}\right|^{2}=\sum_{k=1}^{n}\left(c_{\alpha}^{(k)}\right)^{2}$ if $c_{\alpha}=\left(c_{\alpha}^{(1)}, \ldots, c_{\alpha}^{(n)}\right)$ $\in \mathbb{R}^{n}$, and $\mu$ is the Lévy white noise measure on $\left(\mathcal{S}^{\prime}(\mathbb{R}), \mathcal{B}\left(\mathcal{S}^{\prime}(\mathbb{R})\right)\right)$, $\alpha !=\prod_{k=1}^{\infty} \alpha_{k} !$ and $(2 \mathbb{N})^{\alpha}=\prod_{j}(2 j)^{\alpha}$ for $\alpha \in \mathcal{J}$.

The space $(\mathcal{S})_{-1}^{n}$ consist of all formal expansions $F(\omega)=\sum_{\alpha} b_{\alpha} K_{\alpha}(\omega)$ with $b_{\alpha} \in \mathbb{R}^{n}$ such that $\|F\|_{-1,-q}^{2}=\sum_{\alpha} b_{\alpha}^{2}(2 \mathbb{N})^{-q \alpha}<\infty$ for some $q \in \mathbb{N}$. The family of seminorms $\|f\|_{1, k}, k \in \mathbb{N}$ gives rise to a topology on $(\mathcal{S})_{1}^{n}$, and we can regard $(\mathcal{S})_{-1}^{n}$ as the dual of $(\mathcal{S})_{1}^{n}$ by the action $\langle F, f\rangle=\sum_{\alpha}$ $\left(b_{\alpha}, c_{\alpha}\right) \alpha$ !, where $\left(b_{\alpha}, c_{\alpha}\right)$ is the usual inner product in $\mathbb{R}^{n}$.

The Wick product $F \diamond G$ of two elements $F=\sum_{\alpha} a_{\alpha} K_{\alpha}, G=\sum_{\alpha} b_{\alpha}$ $K_{\alpha} \in(\mathcal{S})_{-1}^{n}$ with $\alpha_{\alpha}, b_{\alpha} \in \mathbb{R}^{n}$, is defined by

$$
F \diamond G=\sum_{\alpha, \beta}\left(a_{\alpha}, b_{\beta}\right) K_{\alpha+\beta} .
$$

One can prove that the spaces $\mathcal{S}\left(\mathbb{R}^{d}\right), \mathcal{S}^{\prime}\left(\mathbb{R}^{d}\right),(\mathcal{S})_{1}$, and $(\mathcal{S})_{-1}$ are closed under Wick product.

For $F=\sum_{\alpha} a_{\alpha} K_{\alpha} \in(\mathcal{S})_{-1}^{n}$ with $a_{\alpha} \in \mathbb{R}^{n}$, the Lévy Hermite transform of $F$, denoted by $\mathcal{H}(F)$ or $\widetilde{F}$, is defined by

$$
\mathcal{H}(F)(z)=\widetilde{F}(z)=\sum_{\alpha} a_{\alpha} z^{\alpha} \in \mathbb{C}^{n} \quad \text { (when convergent) }
$$

where $z=\left(z_{1}, z_{2}, \ldots\right) \in \mathbb{C}^{\mathbb{N}}$ (the set of all sequences of complex numbers) and $z^{\alpha}=z_{1}^{\alpha_{1}} z_{2}^{\alpha_{2}} \ldots z_{n}^{\alpha_{n}} \ldots$ for $\alpha=\left(\alpha_{1}, \alpha_{2}, \ldots\right) \in \mathcal{J}$. 
For $F, G \in(\mathcal{S})_{-1}^{n}$ by this definition, we have

$$
\widetilde{F \diamond G}(z)=\widetilde{F}(z) \cdot \widetilde{G}(z),
$$

for all $z$ such that $\widetilde{F}(z), \widetilde{G}(z)$ exist. The product on the right-hand side of the above formula is the complex bilinear product between two elements of $\mathbb{C}^{\mathbb{N}}$ defined by $\left(z_{1}^{(1)}, \ldots, z_{n}^{(1)}\right) .\left(z_{1}^{(2)}, \ldots, z_{n}^{(2)}\right)=\sum_{k=1}^{n} z_{k}^{(1)} z_{k}^{(2)}$, where $z_{k}^{(i)} \in \mathbb{C}$

Let $F=\sum_{\alpha} a_{\alpha} K_{\alpha} \in(\mathcal{S})_{-1}^{n}$. Then the vector $\widetilde{F}(0) \in \mathbb{R}^{n}$ is called the generalized expectation of $F$ and is denoted by $\mathbb{E}(F)$. Suppose that $X: V \rightarrow \mathbb{C}^{m}$ is an analytic function, where $V$ is a neighbourhood of $\mathbb{E}(F)$. Assume that the Taylor series of $X$ around $\mathbb{E}(F)$ has coefficients in $\mathbb{R}^{n}$. Then the Wick version $X^{\diamond}(F)=\mathcal{H}^{-1}(X \circ \widetilde{F}) \in(\mathcal{S})_{-1}^{m}$.

Suppose that modelling considerations lead us to consider an SPDE expressed formally as $A\left(t, x, \partial_{t}, \nabla_{x}, U, \omega\right)=0$, where $A$ is some given function, $U=U(x, t, \omega)$ is the unknown (generalized) stochastic process, and where the operators $\partial_{t}=\frac{\partial}{\partial t}, \nabla_{x}=\left(\frac{\partial}{\partial x_{1}}, \ldots, \frac{\partial}{\partial x_{d}}\right)$ when $x=\left(x_{1}\right.$, $\left.\ldots, x_{d}\right) \in \mathbb{R}^{d}$. Firstly, we interpret all products as Wick products and all functions as their Wick versions. We indicate this as

$$
A^{\diamond}\left(t, x, \partial_{t}, \nabla_{x}, U, \omega\right)=0 .
$$

Secondly, we take the Lévy Hermite transform of (2.3). This turns Wick products into ordinary products (between complex numbers) and the equation takes the form

$$
\widetilde{A}\left(t, x, \partial_{t}, \nabla_{x}, \tilde{U}, z_{1}, z_{2}, \ldots\right)=0,
$$

where $\tilde{U}=\mathcal{H} U$ is the Lévy Hermite transform of $U$ and $z_{1}, z_{2}, \ldots$ are complex numbers. Suppose we can find a solution $u=u(x, t, z)$ of the 
equation $\tilde{A}\left(t, x, \partial_{t}, \nabla_{x}, u, z\right)=0$, for each $z=\left(z_{1}, z_{2}, \ldots\right) \in \mathbb{V}_{q}(r)$ for some $q, r$, where $\mathbb{V}_{q}(r)=\left\{z=\left(z_{1}, z_{2}, \ldots\right) \in \mathbb{C}^{\mathbb{N}}: \sum_{\alpha \neq 0}\left|z^{\alpha}\right|^{2}(2 \mathbb{N})^{q \alpha}<r^{2}\right\}$. Then, under certain conditions, we can take the inverse Lévy Hermite transform $U=\mathcal{H}^{-1} u \in(\mathcal{S})_{-1}$ and thereby obtain a solution $U$ of the original (Wick) equation (2.3). Sufficient conditions for this procedure to work are given in the next theorem.

Theorem 2.1 ([25, 26]). Suppose $u(x, t, z)$ is a solution of the Lévy Hermite transformed equation (2.4) for $(x, t)$ in some bounded open set $\mathbf{D} \in \mathbb{R}^{d} \times \mathbb{R}_{+}$, and for all $z \in \mathbb{V}_{q}(r)$ some $q$, $r$. Moreover, suppose that $u(x, t, z)$ and all its partial derivatives, which are involved in Equation (2.4), are uniformly bounded for $(x, t, z) \in \mathbf{D} \times \mathbb{V}_{q}(r)$, continuous with respect to $(x, t) \in D$ for all $z \in \mathbb{V}_{q}(r)$ and analytic with respect to $z \in \mathbb{V}_{q}(r)$ for all $(x, t) \in \mathbf{D}$. Then there exists $U(x, t) \in(\mathcal{S})_{-1}$ such that $u(x, t, z)=(\widetilde{U}(x, t))(z)$ for all $(x, t, z) \in \mathbf{D} \times \mathbb{V}_{q}(r)$ and $U(x, t)$ solves Equation (2.3) in $(\mathcal{S})_{-1}$.

\section{Lévy White Noise Functional Solutions for Equation (1.1)}

In this section, first we reduce Equation (1.1) into a deterministic partial differential equation by applying Lévy Hermite transform. Further, by applying proper transformation, the obtained partial differential equation can be converted into a non-linear ordinary differential equation. Then, by employed the proposed $F$-expansion method, we obtain a family of exact solutions for the formulated partial differential equation. Then, by using Theorem 2.1 for $d=1$, we can take the inverse Lévy Hermite transform and thereby obtain Lévy white noise functional solutions of Equation (1.1). 
Taking the Lévy Hermite transform of Equation (1.1), we get the deterministic equation

$\widetilde{U}_{t}(x, t, z)+(f(t)+\alpha \tilde{\dot{\eta}}(t, z)) \tilde{U}(x, t, z) \widetilde{U}_{x}(x, t, z)+(g(t)+\beta \tilde{\dot{\eta}}(t, z)) \widetilde{U}_{x x x}(x, t, z)=0$,

where the Lévy Hermite transform of $\dot{\eta}(t)$ is defined by $\tilde{\dot{\eta}}(t, z)=$ $\left(\int_{\mathbb{R}} \theta^{2} \nu(d \theta)\right)^{1 / 2} \sum_{i=1}^{\infty} \xi_{i}(t) z_{\kappa}(i, 1)$ when $z=\left(z_{1}, z_{2}, \ldots\right) \in\left(\mathbb{C}^{\mathbb{N}}\right)_{c}$ is a vector parameter. For sake of simplicity, let $u(x, t, z)=\tilde{U}(x, t, z), \bar{f}(t, z)=f(t)$ $+\alpha \tilde{\dot{\eta}}(t, z)$ and $\bar{g}(t, z)=g(t)+\beta \tilde{\dot{\eta}}(t, z)$.

We seek the travelling wave solution of Equation (3.1) of the form

$$
u(x, t, z)=\varphi(\zeta(x, t, z)), \zeta(x, t, z)=l(t, z) x+m(t, z),
$$

where $l(t, z), m(t, z)$ are functions to be determined later. Substituting Equation (3.2) into Equation (3.1) yields a non-linear ordinary differential equation for $\varphi($.

$$
\left(l_{t}(t, z) x+m_{t}(t, z)\right) \varphi^{\prime}(\zeta)+\bar{f}(t, z) l(t, z) \varphi(\zeta) \varphi^{\prime}(\zeta)+\bar{g}(t, z) l^{3}(t, z) \varphi^{\prime \prime \prime}(\zeta)=0
$$

In Equation (3.3), considering the homogeneous balance between $\varphi(\zeta) \varphi^{\prime}(\zeta)$ and $\varphi^{\prime \prime \prime}(\zeta)$, we can suppose that $\varphi($.$) can be expressed in the$ following form:

$$
\varphi(\zeta)=A(t, z)+B(t, z) \Phi(\zeta)+C(t, z) \Phi^{2}(\zeta),
$$

where $A(t, z), B(t, z)$, and $C(t, z)$ are functions to be determined later and $\Phi($.$) satisfies the elliptic equation$

$$
\left(\Phi^{\prime}(\zeta)\right)^{2}=c_{0}+c_{1} \Phi^{2}(\zeta)+c_{2} \Phi^{4}(\zeta)
$$

where $c_{0}, c_{1}$, and $c_{2}$ are arbitrary constants. Substituting (3.4) and (3.5) into (3.3) and setting the coefficients of $\Phi^{i}, \Phi^{i} \Phi^{\prime}(i=0,1,2,3)$ to zero, 
respectively, we can deduce the following system of equations in the unknowns $A(t, z), B(t, z), C(t, z), l(t, z)$, and $m(t, z)$.

$$
\begin{gathered}
A_{t}=B_{t}=C_{t}=0, \\
B\left(x l_{t}+m_{t}+\bar{f} A l+\bar{g} l^{3} c_{1}\right)=0, \\
2 C\left(x l_{t}+m_{t}\right)+\bar{f} B^{2} l+8 c_{1} \bar{g} l^{3} C+2 \bar{f} A C l=0, \\
3 B l\left(2 \bar{g} l^{2} c_{2}+\bar{f} C\right)=0, \\
2 C l\left(\bar{f} C+12 \bar{g} l^{2} c_{2}\right)=0 .
\end{gathered}
$$

From Equation (3.6), we have

$$
A(t, z)=\sigma_{0}, \quad B(t, z)=\sigma_{1}, \quad C(t, z)=\sigma_{2},
$$

where $\sigma_{0}, \sigma_{1}$, and $\sigma_{2}$ are arbitrary constants. It follows from Equations (3.7) and (3.8) that

$$
l(t, z)=L,
$$

where $L \neq 0$ is an arbitrary constant. In view of Equations (3.10) and (3.12), we obtain

$$
C(t, z)=-\frac{12 c_{2} L^{2} \bar{g}(t, z)}{\bar{f}(t, z)}
$$

By virtue of Equations (3.11) and (3.13), we deduce that $\bar{f}(t, z)$ and $\bar{g}(t, z)$ are linearly dependant, that is

$$
\bar{g}(t, z)=\gamma_{0} \bar{f}(t, z), \quad \text { for all }(t, z) \in \mathbb{R}_{+} \times\left(\mathbb{C}^{\mathbb{N}}\right)_{c},
$$

where $\gamma_{0}$ is an arbitrary constant. Substituting Equation (3.14) into Equation (3.13), we get

$$
C(t, z)=-12 \gamma_{0} L^{2} c_{2}
$$


According to Equation (3.9), we obtain

$$
B(t, z)=\sigma_{1}=0 .
$$

Inserting Equations (3.12), (3.15), and (3.16) into Equation (3.8), gives

$$
A(t, z)=\sigma_{0}=-\frac{m_{t}(t, z)}{L \bar{f}(t, z)}-4 \gamma_{0} L^{2} c_{1}
$$

Since $\sigma_{0}$ is an arbitrary constant, we can assume that

$$
-\frac{m_{t}(t, z)}{L \bar{f}(t, z)}=\gamma_{1}
$$

where $\gamma_{1}$ is an arbitrary constant. It follows from Equations (3.17) and (3.18) that

$$
m(t, z)=-L \gamma_{1} \int_{0}^{t} \bar{f}(\tau, z) d \tau, \quad A(t, z)=\gamma_{1}-4 \gamma_{0} L^{2} c_{1}
$$

Therefore, we deduce the solution of Equation (3.1) as follows:

$$
u(x, t, z)=\gamma_{1}-4 \gamma_{0} c_{1} L^{2}-12 \gamma_{0} c_{2} L^{2} \Phi^{2}(\zeta(x, t, z)),
$$

with

$$
\zeta(x, t, z)=L\left(x-\gamma_{1} \int_{0}^{t}[f(\tau)+\alpha \tilde{\dot{\eta}}(\tau, z)] d \tau\right)
$$

If we can prove that there exist a bounded open set $D \subset \mathbb{R} \times \mathbb{R}_{+}$, $q>0$ and $r>0$ such that $u(x, t, z), u_{t}(x, t, z), u_{x}(x, t, z)$, and $u_{x x x}(x, t, z)$ are uniformly bounded for $(x, t, z) \in D \times \mathbb{V}_{q}(r)$, continuous with respect to $(x, t) \in D$ for all $z \in \mathbb{V}_{q}(r)$ and analytic with respect to $z \in \mathbb{V}_{q}(r)$ for all $(x, t) \in D$. Theorem 2.1 shows that there exists $U(x, t) \in(\mathcal{S})_{-1} \quad$ such that $\quad u(x, t, z)=\mathcal{H}(U(x, t))(z) \quad$ for $\quad$ all $(x, t, z) \in D \times \mathbb{V}_{q}(r)$ and $U(x, t)$ solves Equation (1.1) in $(\mathcal{S})_{-1}$. From the above, we have that $U(x, t)$ is the inverse Lévy Hermite transform of 
$u(x, t, z)$. Hence, by Equations (3.20) and (3.21), we have the Lévy white noise functional solution of Equation (1.1) as follows:

$$
U(x, t)=\gamma_{1}-4 \gamma_{0} c_{1} L^{2}-12 \gamma_{0} c_{2} L^{2} \Phi^{\diamond 2}(\Theta(x, t)),
$$

with

$$
\Theta(x, t)=L\left(x-\gamma_{1} \alpha \eta(t)-\gamma_{1} \int_{0}^{t} f(\tau) d \tau\right)
$$

In view of the relation $\exp ^{\diamond}(\eta(t))=\exp \left(\eta(t)-t^{2} / 2\right)$ (see [26]), the Lévy white noise functional solution of Equation (1.1) can be given in non-Wick version in the form

$$
U(x, t)=\gamma_{1}-4 \gamma_{0} c_{1} L^{2}-12 \gamma_{0} c_{2} L^{2} \Phi^{2}(\Theta(x, t)),
$$

with

$$
\Theta(x, t)=L\left(x-\gamma_{1} \alpha\left(\eta(t)-\frac{t^{2}}{2}\right)-\gamma_{1} \int_{0}^{t} f(\tau) d \tau\right)
$$

In fact, there are different Jacobi elliptic function solutions for Equation (3.5), these solutions come from setting different values for the parameters $c_{0}, c_{1}$, and $c_{2}$, see Table 1. Therefore, by using Equations (3.24), (3.25) and Table 1, we can get various stochastic Jacobi elliptic function and soliton-like solutions for Equation (1.1). We give two cases as examples:

(I) For $c_{0}=1, c_{1}=-\left(1+m^{2}\right)$, and $c_{2}=m^{2},(0<m<1)$, the solution of Equation (3.5) is $\Phi(\zeta(x, t, z))=\operatorname{sn}(\zeta(x, t, z))$. Thus, the solution of Equation (3.1) is

$$
u_{1}(x, t, z)=\gamma_{1}+4 \gamma_{0} L^{2}\left(1+m^{2}\right)-12 \gamma_{0} L^{2} m^{2} \operatorname{sn}^{2}(\zeta(x, t, z)),
$$

with $\zeta(x, t, z)$ being defined by Equation (3.21). 
Table 1. Some Jacobi elliptic function solutions for Equation (3.5), where $m$ is the Jacobi function module

\begin{tabular}{llll}
\hline$c_{0}$ & $c_{1}$ & $c_{2}$ & $\Phi$ \\
\hline 1 & $-\left(1+m^{2}\right)$ & $m^{2}$ & sn \\
$1-m^{2}$ & $2 m^{2}-1$ & $-m^{2}$ & $\mathrm{cn}$ \\
$m^{2}-1$ & $2-m^{2}$ & -1 & $\mathrm{dn}$ \\
$m^{2}$ & $-\left(1+m^{2}\right)$ & 1 & $\mathrm{~ns}$ \\
$-m^{2}$ & $2 m^{2}-1$ & $1-m^{2}$ & $\mathrm{nc}$ \\
-1 & $2-m^{2}$ & $1-m^{2}$ & $\mathrm{nd}$ \\
1 & $2-m^{2}$ & $1-m^{2}$ & $\mathrm{sc}$ \\
\hline
\end{tabular}

The properties of the Jacobian elliptic function $\operatorname{sn}(x)$ (see Chapter 10 in [32]) shows that the conditions of Theorem 2.1 are all satisfied. Hence, there is a stochastic process $U_{1}(x, t) \in(\mathcal{S})_{-1}$ which is the inverse Lévy Hermite transform of $u_{1}(x, t, z)$. Therefore, we have the following stochastic Jacobi elliptic function solution of Equation (1.1):

$$
U_{1}(x, t)=\gamma_{1}+4 \gamma_{0} L^{2}\left(1+m^{2}\right)-12 \gamma_{0} L^{2} m^{2} \operatorname{sn}^{2}(\Theta(x, t)),
$$

with $\Theta(x, t)$ being defined by Equation (3.25).

In the limit case when $m \rightarrow 1$, we have $\operatorname{sn}(\zeta) \rightarrow \tanh (\zeta)$. Thus, we can get a stochastic soliton-like solution for Equation (1.1) in the form

$$
U_{1}^{*}(x, t)=\gamma_{1}+8 \gamma_{0} L^{2}-12 \gamma_{0} L^{2} \tanh ^{2}(\Theta(x, t)),
$$

where $\Theta(x, t)$ is defined by Equation (3.25).

(II) For $c_{0}=1-m^{2}, c_{1}=2 m^{2}-1$, and $c_{2}=-m^{2},(0<m<1)$, the solution of Equation (3.5) is $\Phi(\zeta(x, t, z))=\operatorname{cn}(\zeta(x, t, z))$. Thus, the solution of Equation (3.1) is

$$
u_{2}(x, t, z)=\gamma_{1}-4 \gamma_{0} L^{2}\left(2 m^{2}-1\right)+12 \gamma_{0} L^{2} m^{2} \operatorname{cn}^{2}(\zeta(x, t, z)),
$$

with $\zeta(x, t, z)$ being defined by Equation (3.21). 
The properties of the Jacobian elliptic function $\operatorname{cn}(x)$ (see Chapter 10 in [32]) shows that the conditions of Theorem 2.1 are all satisfied. Hence, there is a stochastic process $U_{2}(x, t) \in(\mathcal{S})_{-1}$ which is the inverse Lévy Hermite transform of $u_{2}(x, t, z)$. Therefore, we have the following stochastic Jacobi elliptic function solution of Equation (1.1):

$$
U_{2}(x, t)=\gamma_{1}-4 \gamma_{0} L^{2}\left(2 m^{2}-1\right)+12 \gamma_{0} L^{2} m^{2} \operatorname{cn}^{2}(\Theta(x, t)),
$$

where $\Theta(x, t)$ is defined by Equation (3.25).

For $m \rightarrow 1$, we have $\operatorname{cn}(\zeta) \rightarrow \operatorname{sech}(\zeta)$. Thus, Equation (3.30) gives a stochastic soliton-like solution for Equation (1.1) in the form

$$
U_{2}^{*}(x, t)=\gamma_{1}-4 \gamma_{0} L^{2}+12 \gamma_{0} L^{2} \operatorname{sech}^{2}(\Theta(x, t)),
$$

with $\Theta(x, t)$ which is defined by Equation (3.25).

The behaviours of the obtained solutions in Equations (3.28) and (3.31) are shown graphically in Figure 1 and 2 for given parameters. Figure 1 represents the evolutional behaviours of the solution in Equation (3.28) with and without effect of Lévy white noise. Also, Figure 2 presents the behaviours of the solution in Equation (3.31) with and without effect of Lévy white noise. Hence, it is concluded that the stochastic forcing term leads to the uncertainty of the wave amplitude. 


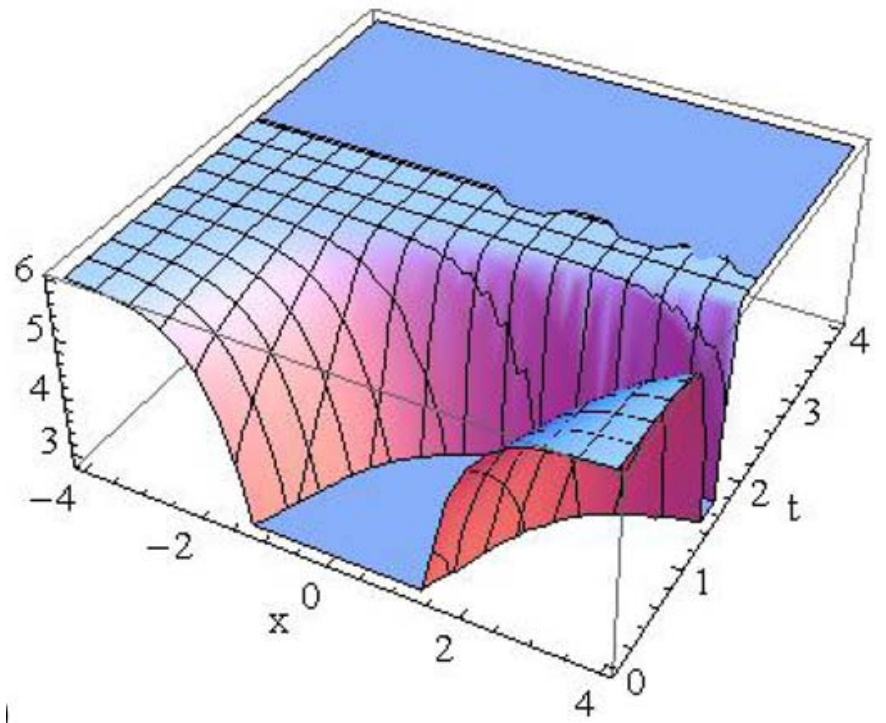

(a)

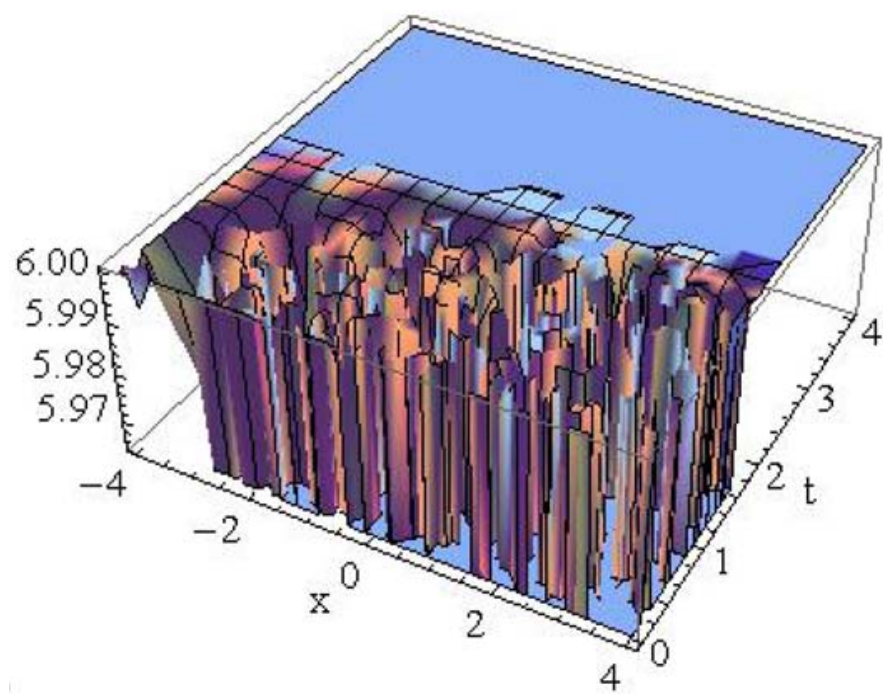

(b)

Figure 1. (a) shows the behaviour of the solution $U_{1}^{*}(x, t)$ without the effect of Lévy white noise. (b) shows the effect of Lévy white noise on the solution $U_{1}^{*}(x, t)$. Here, $\gamma_{0}=L=-1, \gamma_{1}=2, \alpha=0.5$ and $f(t)=\exp \left(t^{2}\right) / 2$. 


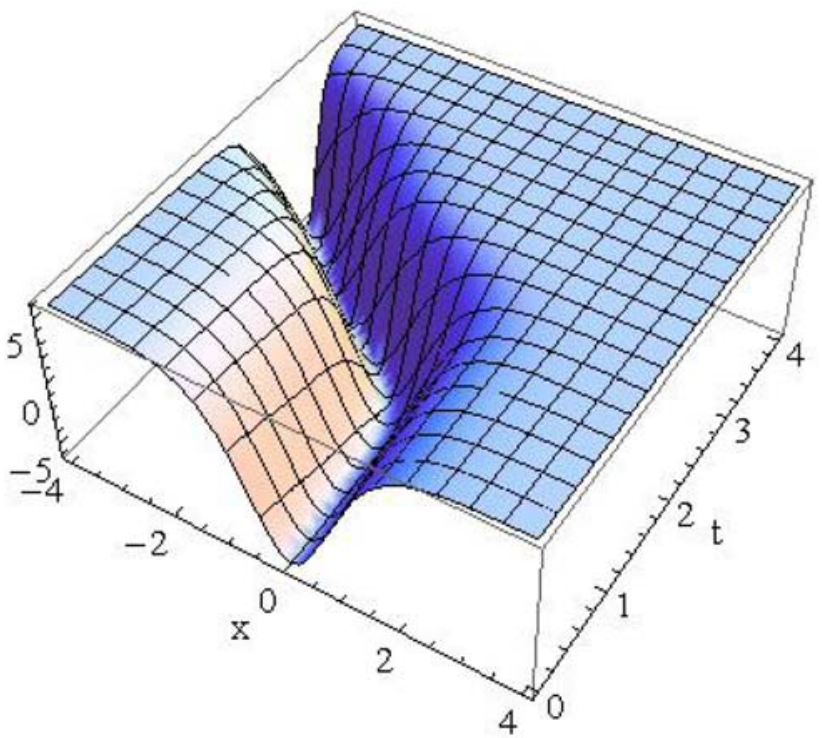

(a)

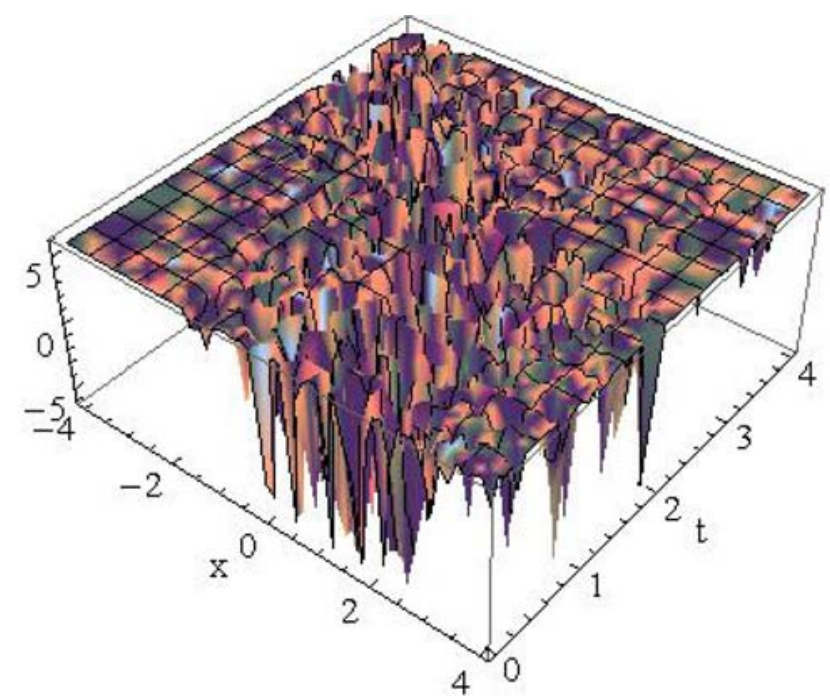

(b)

Figure 2. (a) shows the behaviour of the solution $U_{2}^{*}(x, t)$ without the effect of Lévy white noise. (b) shows the effect of Lévy white noise on the solution $U_{2}^{*}(x, t)$. Here, $\gamma_{0}=L=-1, \gamma_{1}=2, \alpha=0.5$ and $f(t)=\sin \left(t^{2}\right) / 2$. 
Remark. In fact, if we impose the condition that a Lévy process $\eta(t)$ is continuous, then it has the form

$$
\eta(t)=a t+\sigma B(t)
$$

where $a$ and $\sigma$ are constants and $B(t)$ is the real Brownian motion, so we are basically back to the Gaussian case. Therefore, we have a new white noise functional solutions of the Gaussian white noise Wick-type stochastic KdV equation. These solutions can be give, by using Equations (3.24), (3.25), and (3.32), in the form

$$
U(x, t)=\gamma_{1}-4 \gamma_{0} c_{1} L^{2}-12 \gamma_{0} c_{2} L^{2} \Phi^{2}(\Theta(x, t)),
$$

with

$$
\Theta(x, t)=L\left(x-\gamma_{1} \alpha\left(a t+\sigma B(t)-\frac{t^{2}}{2}\right)-\gamma_{1} \int_{0}^{t} f(\tau) d \tau\right)
$$

where $\Phi($.$) runs through all Jacobi elliptic function solutions of Equation$ (3.5).

\section{Conclusion}

This paper is devoted to use Lévy white noise setting, in particular, Lévy Wick product, Hermite transform and Kondratiev spaces to present a new approach to study the non-linear stochastic partial differential equations driven by Lévy white noise. Then, by using this approach and the $F$-expansion method, many exact stochastic Jacobi elliptic function and soliton-like solutions for the Lévy Wick-type stochastic KdV equation are showed. Obviously, the planner which we have proposed in this paper can be also applied to other non-linear partial differential equations in mathematical physics such as KdV-Burgers, modified KdV-Burgers, Sawada-Kotera, Zhiber-Shabat, and Benjamin-Bona-Mahony equations. Moreover, since $\Psi^{\diamond}()=.\Psi($.$) for any non-random function \Psi($.$) ,$ 
Equations (3.24) and (3.25) give a new set of exact solutions of the variable coefficients $\mathrm{KdV}$ equation (1.2), where $\Phi($.$) runs through all$ Jacobi elliptic function solutions of Equation (3.5).

\section{References}

[1] M. Wadati, Stochastic Korteweg-de Vries equation, J. Phys. Soc. Jpn. 52 (1983), 2642-2648.

[2] Y. C. Xie, Exact solutions for stochastic KdV equations, Phys. Lett. A 310 (2003), 161-167.

[3] B. Chen and Y. C. Xie, Exact solutions for Wick-type stochastic coupled KadomtsevPetviashili equations, J. Phys. A: Math. Gen. 38 (2005), 815-822.

[4] B. Chen and Y. C. Xie, Exact solutions for generalized stochastic Wick-type KdV-mKdV equations, Chaos, Solitons and Fractals 23 (2005), 281-288.

[5] B. Chen and Y. C. Xie, White noise functional solutions of Wick-type stochastic generalized Hirota-Satsuma coupled KdV equations, J. Comput. Appl. Math. 197 (2006), 345-354.

[6] B. Chen and Y. C. Xie, Periodic-like solutions of variable coefficients and Wick-type stochastic NLS equations, 203 (2007), 249-263.

[7] Y. C. Xie, An auto-Bäcklund transformation and exact solutions for Wick-type stochastic generalized KdV equations, J. Phys. A: Math. Gen. 37 (2004), 5229-5236.

[8] Y. C. Xie, Positonic solutions for Wick-type stochastic KdV equations, Chaos, Solitons and Fractals 20 (2004), 337-342.

[9] Y. C. Xie, Exact solutions of the Wick-type stochastic Kadomtsev-Peviashvili equations, Chaos, Solitons and Fractals 21 (2004), 473-480.

[10] H. A. Ghany. Exact solutions for stochastic generalized Hirota-Satsuma coupled KdV equations. Chin. J. Phys. 49 (2011), 926-940.

[11] H. A. Ghany, Exact solutions for KdV-Burger equations with an application of whitenoise analysis, International Journal of Pure and Applied Mathematics 78 (2012), 17-27.

[12] H. A. Ghany, Analytical approach to exact solutions for the Wick-type stochastic space-time fractional KdV equation, Chin. Phys. Lett. 31 (2014), 060503.

[13] H. A. Ghany, A. S. Okb El Bab, A. M. Zabal and A. Hyder, The fractional coupled $\mathrm{KdV}$ equations: Exact solutions and white noise functional approach, Chin. Phys. B 22 (2013), 0805011. 
[14] H. A. Ghany and A. Hyder, White noise functional solutions for Wick-type stochastic coupled KdV equations, Journal of Mathematical Sciences: Advances and Applications 11 (2011), 79-96.

[15] H. A. Ghany and A. Hyder, White noise functional solutions for the Wick-type twodimensional stochastic Zakharov-Kuznetsov equations, Int. Rev. Phys. 6 (2012), 153-157.

[16] H. A. Ghany and A. Hyder, Local and global well-posedness of stochastic ZakharovKuznetsov equation, J. Comput. Anal. Appl. 15 (2013), 1332-1343.

[17] H. A. Ghany and A. Hyder, Exact solutions for the Wick-type stochastic timefractional KdV equations, Kuwait Journal of Science 41 (2014), 75-84.

[18] H. A. Ghany and A. Hyder, Abundant solutions of Wick-type stochastic fractional 2D KdV equation, Chin. Phys. B 23 (2014), 060503.

[19] H. A. Ghany, A. Hyder and M. Zakarya, Non-Gaussian white noise functional solutions of $\chi$-Wick-type stochastic KdV equations, Appl. Math. Inf. Sci., Accepted (2017).

[20] A. Hyder and M. Zakarya, Non-Gaussian Wick calculus based on hypercomplex systems, International Journal of Pure and Applied Mathematics 109 (2016), 539-556.

[21] S. Albeverio, Yu. G. Kondratev and L. Streit, How to generalize white noise analysis to non-Gaussian spaces, in: Dynamics of Complex and Irregular Systems, World Scientific, Singapore (1993), 48-60.

[22] Y. Kondratiev, J. L. DA Silva and L. Streit, Generalized Appell system, Meth. Funct. Anal. Topol. 3 (1997), 28-61.

[23] Y. Kondratiev, J. L. DA Silva, L. Streit and G. US, Analysis on Poisson and gamma spaces, Infin. Dim. Anal. Quantum Probab. Relat. Top. 1 (1998), 91-117.

[24] B. A. Løkka, B. Øksendal and F. Proske, Stochastic partial differential equations driven by Lévy space-time white noise, The Annals of Applied Probability 14 (2004), 1506-1528.

[25] B. Øksendal, Stochastic partial differential equations driven by multi-parameter white noise of Lévy processes, Quart. Appl. Math. 66 (2008), 521-537.

[26] H. Holden, B. Øksendal, J. Ubøe and T. Zhang, Stochastic Partial Differential Equations, Springer Science+Business Media, LLC, 2010.

[27] A. de Bouard and A. Debussche, On the stochastic Korteweg-de Vries Equation, J. Funct. Anal. 154 (1998), 215-251.

[28] M. L. Wang, Y. M. Wang, A new Bäcklund transformation and multi-soliton solutions to the KdV equation with general variable coefficients, Phys. Lett. A 287 (2001), 211-216.

[29] C. S. Gardner, J. M. Greene, M. D. Kruskal and R. M. Miura, Method for solving the KdV equation, Phys. Rev. Lett. 19 (1967), 1095-1097. 
[30] S. K. Liu, Q. Zhao, Z. T. Fu and S. D. Liu, Expansion method about the Jacobi elliptic function and its applications to nonlinear wave equations, Acta Phys. Sin. 50 (2001), 2068-2073.

[31] B. Øksendal and F. Proske, White noise of Poisson random measures, Potential Anal. 21 (2004), 375-403.

[32] Z. X. Wang and D. R. Guo, Introduction to Special Functions, Peking University Press: China, 2000. 